\title{
Involvement of p67 in Actin Cytoskeleton Dynamics, Survival, Migration, and Motility during Differentiation of C2C12 Myoblasts into Multinucleated Myotubes
}

\author{
Bansidhar Datta \\ Department of Chemistry \& Biochemistry, Kent State University, Kent, OH 44242 \\ bdatta@kent.edu
}

\begin{abstract}
Summary: During wound healing, myogenic satellite cells or myoblasts fuse within the damaged myotubes in the presence of serum. Before the fusion, myoblasts undergo a series of steps including multiplication to a greater number, shutting off the growth-promoting signals, expressing myogenic proteins, degrading myogenic inhibitors, and upregulating the expression of other muscle-specific proteins that are involved in the survival, migration, alignment, and fusion into myotubes. During differentiation of C2C12 myoblasts into multinucleated myotubes, the expression of eukaryotic initiation factor 2 (eIF2)-associated glycoprotein p67, increases and its level maximizes at the time of myoblasts fusion into myotubes. During this time of differentiation, p67 dissociates from eIF2 and associates with extracellular signal-regulated kinases 1 and 2 (ERK1/2). Dissociation from eIF2 leads to high levels of phosphorylation of smallest $\alpha$-subunit of eIF2 that suppresses the rates of global protein synthesis. Association of p67 with ERK1/2 kinases leads to the inhibition of activation and activity of the later kinases and subsequently this causes the shutting off the growth-promoting signal to myoblasts. P67 has intermolecular and intra-molecular proteolysis activity. This activity is not only involved in the degradation of certain cell cycle regulatory proteins, cyclins and Cdks both in myoblasts and in myotubes, it is also involved in the degradation of potent growth-promoting proteins like, myristoylated alanine-rich $C$ kinase substrate (MARCKS) and focal adhesion kinase (FAK). By downregulating the expression and activity of the later proteins, p67 possibly enhances myoblasts' actin cytoskeleton dynamics, migration, and alignment before fusion into myotubes. In contrast, p67 is also involved in the upregulated expression and activity of cPKC $\alpha$ that has the ability to fuse myoblasts into myotubes in the presence of serum. In addition, increased expression of p67 during differentiation is involved in the upregulated expression and activity of p21-activating kinase 1 (Pakl), which is required for the motility and glucose homeostasis of skeletal muscles both in murine and human. Altogether, our findings suggest that p67 is involved in actin cytoskeleton dynamics, survival, migration, and alignment of myoblasts while fusing into multinucleated myotubes.
\end{abstract}

Abbreviations used: $p 67$, an eukaryotic initiation factor 2 (eIF2)-associated glycoprotein; eIF $2 \alpha$, the smallest $\alpha$-subunit of eIF2; ERK1/2, extracellular signal-regulated kinases; MEK1/2; mitogen-activated protein kinase kinase 1 and 2; Cdks, cyclin-dependent kinases; MARCKS, myristoylated-alanine rich C kinase substrate; FAK, focal adhesion kinase; Pak1, p21-activating kinase 1; cPKC $\alpha$, conventional protein kinase C $\alpha$; Akt/PKB, protein kinase B; ECM, extracellular matrix; RTK, receptor tyrosine kinase; PLC $\gamma$, phospholipase C $\gamma$; DAG, diacylglycerol; SH2 and SH3, Src homology domain 2 and 3; Cdc42, a Rho GTPase; p52, the downstream 108480 amino acid segment of p67; and MEF2, myogenic enhancer factor 2.

Keywords: eIF2-associated glycoprotein p67, MARCKS, FAK, Pak1, PKC, C2C12 myoblasts differentiation, actin cytoskeleton dynamics, cell migration, cell motility, and myoblasts fusion.

\section{INTRODUCTION}

Skeletal muscle differentiation into multinucleated myotubes is a highly orchestrated cellular process that includes a series of steps (1). These steps include but not limited to (i) inhibition of growth promoting signals including suppression of global protein synthesis to withdraw myoblasts permanently from cell cycle (2-3), (ii) expression of muscle-specific proteins like myogenic family of proteins and degradation of myogenic inhibitors (1), (iii) detachment of anchored myoblasts from extracellular matrix (ECM) (4), (iv) survival, migration and alignments of myoblasts (5-7), (v) regulation of actin cytoskeleton dynamics to form filopodia and lamillopodia (8-9), (vi) fusions of myoblasts into multinucleated myotubes (10-11), (vii) $\mathrm{Ca}^{2+}$ homeostasis (12), (viii) promotion of motility by preventing apoptosis (13-14), and finally (viii) formation of myofibers for contractile apparatus (15). Many of these steps are also involved during regeneration, repair, and wound healing 
(16-18). Several groups of proteins are involved in these multi-step events (1-3). Among these group of proteins, the mitogen-activated protein (MAP) kinases, extracellular signal-regulated kinases 1 \& 2 (ERK1/2), the integrin-mediated adhesion to extracellular matrix (ECM) proteins, lipid induced protein kinase $\mathrm{C}(\mathrm{PKC})$, and several others control proliferation of myogenic cells $(2-3,5,19-22)$. When needed these growth promoting signaling pathways shut off and myoblasts start to differentiate into myotubes.

\section{P67 INHIBITS THE RATES OF GLOBAL PROTEIN SYNTHESIS AND ERK1/2 MAPK- MEDIATED SIGNALING DURING DIFFERENTIATION OF C2C12 MYOBLASTS INTO MYOTUBES}

A cellular glycoprotein p67, which binds to both eukaryotic initiation factor 2 (eIF2) and ERK1/2 MAP kinases, modulates their activities (23-24). During normal cell growth, it is bound to eIF2 in 1:1 molar ratio and regulates the level of phosphorylation of smallest $\alpha$-subunit of eIF2 (eIF2 $\alpha$ ) (25). Once p67 dissociates from eIF2, eIF2 $\alpha$ is phosphorylated (25-26) and higher level of eIF2 $\alpha$ phosphorylation is inhibitory to the rates of global protein synthesis (27-30). When level of p67 is increased inside cells due to its low turn over rate (31) or some other condition like differentiation of C2C12 myoblasts into myotubes (32), it associates with ERK1/2 kinases and inhibits its MAP kinase kinase (MEK1/2)-mediated phosphorylation (31-32). This then inhibits the ERK1/2-mediated signaling to the nucleus and suppresses the cell cycle progression (33-35). Indeed, the level of p67 increases gradually during differentiation of $\mathrm{C} 2 \mathrm{C} 12$ myoblasts and its level peaks at the time of fusion into myotubes (32). During this time of differentiation, p67 dissociates from eIF2 leading to the higher levels of phosphorylation of eIF $2 \alpha$ and subsequent suppression of rates of global protein synthesis (26). At the same time, p67 also associates with ERK1/2 and its association maximizes at the time of $\mathrm{C} 2 \mathrm{C} 12$ myoblasts' fusion into myotubes (31). During differentiation of myoblasts into myotubes, there is a permanent withdrawal from the cell cycle leading to total inhibition of DNA synthesis while limited level of transcription and translation of muscle-specific genes stay on (1). In addition, there is a suppression of global transcriptional and translational machineries along with the inhibitions of the growth-promoting signals during myogenic differentiation (2-3). Once the myoblasts receive the signals from these steps they detach from the ECM (4), propagate signals for survival, migration, alignment, and finally fusion into multinucleated myotubes (5-7).

In myofibers, there is a very small population of satellite muscle cells, which are mononuclear progenitor cells residing in a quiescent state between the basement membrane and the sarcolemma of muscle fibers and their number increases in response to ECM-bound hepatocyte growth factor (HGF) and inflammatory stimuli during repair and wound healing (16-18). Once there is a sufficient number of myoblasts, they align on exiting myotubes and ultimately fuse within them (16). In cycling myoblasts growth promoting factors provide signals for proliferation by activating several signaling pathways including mitogen-activated protein kinase (MAPK) cascade, the phospholipase C $\gamma$ (PLC $\gamma$ ) pathway, integrin/FAK (focal adhesive kinase) pathway, the lipid kinase phosphatidylinositol 3 kinase (PI3K) pathway $(2-3,5,19-22)$, and possibly others. These signaling pathways provide potent proliferative signals to myoblasts.

Upon binding to growth factors, receptor tyrosine kinases (RTKs) form dimer that leads to auto- and trans-phosphorylation at their tyrosine residues. The Src-homology domain 2 (SH2) of adapter protein, Grb2 (growth factor receptor-bound protein 2), binds to the phospho-tyrosine residue of RTKs and its SH3 domain binds to the proline-rich region of GDP/GTP exchange factor, SOS (son of seven-less homolog) and activates it. The activated SOS then displaces GDP with GTP from the farnesylated membrane-bound Ras. The membrane-bound Ras-GTP is the active form of Ras that activates the serine/threonine kinase, Raf by phosphorylating it. The signal then propagates to MEK1/2, the downstream effector of phospho-Raf. Subsequently, the phosphorylated MEK1/2 send the signal to ERK1/2 MAP kinases by phosphorylating at their threonine/tyrosine residues and ultimately, the phosphorylated forms of ERK1/2 kinases translocate into the nucleus to turn on the expression of several early genes including cell cycle proteins, cyclin D's that drive the cell cycle into the $\mathrm{G}_{1}$-phase. In the end, this leads to the cell division (36-37). P67 turns off this growth-promoting signal during fusion of myoblasts into myotubes (32). 
Involvement of p67 in Actin Cytoskeleton Dynamics, Survival, Migration, and Motility during Differentiation of C2C12 Myoblasts into Multinucleated Myotubes

\section{OVEREXPRESSION OF P67 DEGRADES CERTAIN CYClINS AND CDKS IN C2C12 MYOBLASTS AND POSSIBLY IN MULTINUCLEATED MYOTUBES}

In eukaryotic cells in general, cell cycle stays on for DNA synthesis to continue. A group of cyclins (cyclins D's, cyclin E, cyclin A, and cyclin B1) and cyclin-dependent kinases (Cdk1, Cdk2, Cdk4, and Cdk6) form various complexes that play most important roles in regulating the different phases of cell cycle (38-39). Usually, the transition from the $\mathrm{G}_{0}$ to $\mathrm{G}_{1}$-phase and maintenance of cells at this phase are regulated by cyclins D's/Cdk4, 6 complexes. The cyclin E/Cdk2 complex ensures the transition to S-phase (DNA synthesis phase) from $\mathrm{G}_{1}$-phase, while cyclin $\mathrm{A} / \mathrm{Cdk} 2$ and in some extend cyclin $\mathrm{E} / \mathrm{Cdk} 2$ complexes maintain proper duplication of $2 \mathrm{n}$ chromosomes to $4 \mathrm{n}$ chromosomes and advancement of cells into the $\mathrm{G}_{2}$-phase of cell cycle. The cyclin B1/Cdk1 complex not only maintains the later stages of $\mathrm{G}_{2}$-phase, it also regulates the $\mathrm{G}_{2} / \mathrm{M}$-phase transition and maintains the M-phase of the cell cycle. Once cell divides into two, it may enter into the quiescence stage by permanently withdrawing from the cell cycle or repeat all the steps of the cell cycle. During the transition from one phase of the cell cycle to the next, most significant degradation of cyclins and in some extend degradation of Cdks are essential (38-39). These degradations may take place either in the cytoplasmic and/or nuclear proteasomes or by some cellular/nuclear proteinases (40). In addition, permanent withdrawal of cells from cell cycle requires complete suppression of expression of several cyclin/Cdk complexes. Multinucleated myotubes are in quiescence stage after permanently withdrawing from cell cycle (16-18).

P67's roles are not limited to the inhibition of ERK1/2-mediated signaling and suppression of rates of global protein synthesis in differentiating $\mathrm{C} 2 \mathrm{C} 12$ myoblasts it is also involved in degradation of certain cyclins and Cdks in these cells (41). Our recent findings suggest that p67 is involved in lowering the overall concentration of cyclin D1/Cdk4 and cyclin D1/Cdk6 complexes and thus inhibits the cell cycle at the $\mathrm{G}_{1}$-phase. It is also involved in proteolytic processing of pro-forms of cyclins E, A, and B1 but not cyclin D1 to maturation. P67 may be involved in lowering the level of Cdk2 possibly by proteolytic degradation and increasing the level of Cdk1 by an unknown mechanism. P67 is also involved in the increased level of cyclin A possibly to show positive outcome during DNA synthesis. It also showed strong effects on degradation of cyclin B1 possibly due to their direct or indirect binding - indicating p67's involvement in the inhibition of $\mathrm{G}_{2}$-phase and $\mathrm{G}_{2} / \mathrm{M}$-phase transition (41). Altogether, our data indicate that increased level of p67 during myoblasts' differentiation into myotubes is possibly to inhibit cell cycle at the $G_{1}$-phase, $G_{1} / S$-phase transition, $\mathrm{G}_{2}$-phase, and $\mathrm{G}_{2} / \mathrm{M}$-phase transition.

\section{OVEREXPRESSION OF P67 IN C2C12 MYOBLASTS INDUCES DIFFERENTIATION BY INCREASING THE LEVELS OF CPKC $\alpha$}

In addition to the MAPK-mediated signaling pathways, other membrane-bound receptors including RTKs are phosphorylated at their tyrosine residues in response to external stimulators, including growth factors, hormones, and other membrane receptor ligands (37). PLC $\gamma$ is phosphorylated at its tyrosine residue upon binding to phospho-tyrosine residue of RTK and this along with translocation to the plasma membrane result in activation of the enzyme. Active PLC $\gamma$ hydrolyzes phosphatidylinositol 4, 5 bis-phosphate $\left(\mathrm{PIP}_{2}\right)$ to generate diacylglycerol (DAG) and inositol 1, 4, 5 triphosphate $\left(\mathrm{IP}_{3}\right)$. The later second messenger binds to certain receptors on the membrane of the endoplasmic reticulum and this releases free cytosolic $\mathrm{Ca}^{2+}$. Protein kinase $\mathrm{C}(\mathrm{PKC})$, a family of phospholipid-dependent serine/threonine kinases has several isozymes such as cPKCs $(\alpha, \beta \mathrm{I}, \beta \mathrm{II}$, and $\gamma)$, nPKCs $(\delta, \varepsilon, \eta$, and $\theta)$, and aPKCs $(\zeta, \mathfrak{l}$, and $\lambda$ ). All isozymes need phosphatidylserine (PS) for activation, whereas certain PKC isozymes need additional co-factors such as diacylglycerol (DAG) and calcium $\left(\mathrm{Ca}^{2+}\right)$ for activation (42). The activated PKC isozymes phosphorylate a series of target proteins and are involved in multiple signal transduction systems that lead to cell growth and proliferation, differentiation, survival, invasion, migration, angiogenesis, apoptosis, and anticancer drug resistance through their increased or decreased participation in various signaling pathways (4344). Due to their involvement in diverse cellular functions, different PKC isozymes are involved in different types of cancers including bladder cancer, breast cancer, colon cancer, gastric cancer, gastrointestinal stromal tumor (GIST), glioma, head and neck cancer, liver cancer, lung cancer, melanoma, myeloid and lymphocytic leukemia, ovarian cancer, pancreatic cancer, prostate cancer, renal cell carcinoma, and thyroid cancer (43-44). Detection of overexpressed or hyperactivated form 


\section{Bansidhar Datta}

of certain PKC isozymes has been used as diagnostic or prognostic biomarkers (43). Overexpression of PKC biomarkers is closely related to poor prognosis, poor response to chemotherapy, and poor survival (43, 45-49). Among the PKC isozymes, cPKC $\alpha$ seems to be involved in almost all types of cancer including breast cancer. In breast cancer, it is highly overexpressed and this leads to poor prognosis and survival because of a deceased response to chemotherapy and the high aggressiveness of the cancer (45-49).

Roles of PKC isozymes in skeletal muscle differentiation are quite intriguing. For example, insulininduced differentiation of $\mathrm{C} 2 \mathrm{C} 12$ myoblasts showed no significant increase of $\mathrm{cPKC} \alpha$ level while level of cPKC $\beta$ I decreased gradually and levels of $\mathrm{cPKC} \beta \mathrm{II}$ and $\mathrm{cPKC} \gamma$ were undetectable in myoblasts and differentiated myotubes. On the other hand, during similar conditions of $\mathrm{C} 2 \mathrm{C} 12$ myoblasts' differentiation, the level of $\mathrm{nPKC} \delta$ decreased gradually while levels of $\mathrm{nPKC} \varepsilon$ and $\mathrm{nPKC} \eta$ increased significantly and there was a low level increase of $\mathrm{nPKC} \theta$ (20). In addition, overexpression of $\mathrm{nPKC} \delta$ promotes proliferation and induces malignant transformation of skeletal muscle cells (50). Likewise, overexpression of $\mathrm{cPKC} \alpha$ promotes while $\mathrm{nPKC} \delta$ inhibits the differentiation of $\mathrm{C} 2 \mathrm{C} 12$ myoblasts into myotubes $(22,50-52)$. Overexpression of $\mathrm{cPKC} \alpha$ in $\mathrm{C} 2 \mathrm{C} 12$ myoblasts induces differentiation into myotubes even in the presence of serum by phosphorylating at Ser-162 site of serum response factor (SRF). The phosphorylated SRF cannot bind to the serum response elements (SRE) of a diverse set of genes, e. g. the immediate early genes c-fos, egr-1, and nur77, and the muscle-specific genes $\alpha$-actin, $\alpha$-myosin heavy chain, and muscle creatine kinase (53-54). Atypical PKCs $(\zeta$ and/or $\lambda$ ) show strong effect on inhibition of myogenic differentiation of satellite cells (MM14) and this inhibition is mediated by Ha-Ras, but independent of ERK1/2 MAP kinases (55).

Recently, we examined the effects of expression of $\mathrm{cPKC} \alpha$ in $\mathrm{C} 2 \mathrm{C} 12$ myoblasts constitutively expressing rat $\mathrm{p} 67$. We found $\sim 10$-fold increase in $\mathrm{cPKC} \alpha$ level in p67-overexpressing myoblasts and p67's N-terminal lysine-rich domains I \& II and in some extend its acidic residue-rich domain are essential for this activity. Although, p67's conserved D251 residue alone has no effect of cPKC $\alpha$ level, this residue is however needed to cooperate with the function(s) of lysine-rich domains I \& II (Fig. 1). The later cooperation was prominent in differentiated myoblasts where cPKC $\alpha$ level was down in p67-overexpressing myotubes. In addition, p67's another conserved residue H331 also cooperates with the functions lysine-rich domains I \& II in lowering the level of cPKC $\alpha$ in $\mathrm{C} 2 \mathrm{C} 12$ differentiated myotubes (Fig. 2). Overexpression of $\mathrm{cPKC} \alpha$ in $\mathrm{C} 2 \mathrm{C} 12$ myoblasts causes differentiation into myotubes even in the presence of serum, which provides growth-promoting signal (53-54). Therefore, our results suggest that overexpression of rat p67 in $\mathrm{C} 2 \mathrm{C} 12$ myoblasts leads to the early differentiation by increasing the levels of $\mathrm{cPKC} \alpha$. Once myoblasts are differentiated into myotubes in differentiation medium, there is no need cPKC $\alpha$ and that's why its level did not show any significant change in rat p67-expressing myotubes.

\section{P67 IS INVOLVED IN THE MODULATION OF ACTIN CYTOSKELETON DYNAMICS AND MIGRATION DURING DIFFERENTIATION OF C2C12 MYOBLASTS INTO MYOTUBES BY MODULATING THE EXPRESSION AND ACTIVITY OF MARCKS}

PKC isozymes phosphorylate various substrates - many of these are specific to certain isozymes (43). Among these substrates, MARCKS is however phosphorylated by all three classes (conventional, novel, and atypical) of PKC isozymes (56-58). In response to activated PKC isozymes, MARCKS is highly phosphorylated at Ser-45, Ser-80, Ser-99, and Ser-116 in bovine and Ser-152, Ser156, and Ser163 in murine (57-58). Phosphorylated active MARCKS cannot translocate into the membrane and thus it prevents from actin binding. Therefore during myogenesis, MARCKS's degradation is essential and $\mathrm{Ca}^{2+}$-dependent proteinase, calpain has been shown to be involved in this process (59$60)$.

Myristoylated alanine-rich $\mathrm{C}$ kinase substrate (MARCKS), which is an actin-binding protein, regulates the cytoskeleton dynamics by crosslinking the actin filaments in the plasma membrane dynamics and migration and motility of myoblasts during their fusion into multinucleated myotubes (61, 59-60, 62-64). Proteolysis of MARCKS by calpain and possibly other proteinases activates its actin-binding activity that modulates actin dynamics and cell migration (60-64). This proteolysis seems to be cPKC $\alpha$ dependent (65) because MARCKS is one of the major substrates of PKC, which when phosphorylates this substrate, inhibits its membrane attachment $(56,65)$. During myogenesis, 
the phosphorylation of MARCKS declines and this correlates with its decreased level in the cytosol (61). In addition, the low level of phosphorylation of MARCKS is closely associated with the membrane fusion of myogenic cells (64).

To examine whether p67 has any role(s) in actin cytoskeleton dynamic and migration of myoblasts when fusing into myotubes, we investigated the levels of myristoylated-alanine rich $\mathrm{C}$ kinase substrate (MARCKS) in C2C12 myoblasts cell lines constitutively expressing rat p67 and some of its mutants. We found that p67 is involved in high levels of expression of MARCKS in myoblasts and its $\mathrm{N}$-terminal lysine-rich domains I \& II are required for such activity. On the other hand, in myotubes constitutively expressing rat p67 the level of MARCKS is very low and p67's N-terminal lysine-rich domains I \& II along with conserved D251 and H331 residues play important roles in this process. In addition, MARCKS is degraded in rat p67-expressing C2C12 myoblasts and p67's conserved D251 residue and lysine residue-rich domains I \& II are involved in this degradation. Altogether, our data suggest that p67 is possibly involved in the inhibition of myoblasts' migration while growing in growth medium, whereas it promotes myoblasts' migration and motility when myoblasts are fusing into myotubes by modulating the expression and activity of MARCKS (66).

\section{P67 IS INVOLVED IN THE MODULATION OF ACTIN CYTOSKELETON DYNAMICS AND MOTILITY OF C2C12 MYOBLASTS DIFFERENTIATING INTO MYOTUBES BY REGULATING THE EXPRESSION AND ACTIVITY OF PAK1}

In addition to MARCKS, several other groups of proteins also regulate cytoskeleton dynamics. One such group is the p21-activating kinases (Pak) (67). Pak family of serine/threonine kinases are effectors for the Rho GTPases Cdc42 and Rac, which act as molecular switches controlling cytoskeletal remodeling, gene expression, cell proliferation, invasion, differentiation, and apoptosis (68). Major function of Paks is to control the cytoskeleton dynamics primarily through the regulation of polymerized actin structures, particularly the formation of filopodia and lamillopodia, consequently affecting the cell shape, motility, and adhesion (69). Group A Paks (Pak1, 2, 3) phosphorylates the regulatory myosin light chain VI, which is involved in membrane trafficking and cell migration (70). The ARP2/3 complex controls actin nucleation and branching. Phosphorylation of the p41-ARC subunit of ARP2/3 complex by Pak1 stimulates the assembly of the cellular cortex of migrating cells (69). Paks also play important roles in cell motility by regulating the leading edge microtubule dynamics (69). High levels of Pak1 in cells result in increased cell motility (71). The Pak1 knockout mice are deficit in glucose homeostasis, which includes inefficient insulin secretion and abnormal glucose clearance. This is reminiscent of the biological role of Pak1 in humans, where Pak1 levels are reduced in type 2 diabetic islets (72). These observations implicate Pak1's direct role in the control of glucose metabolism.

Recently, we examined the expression of Pak1 in C2C12 myoblasts differentiating into myotubes and effects of overexpression of rat p67 and some of its mutants in $\mathrm{C} 2 \mathrm{C} 12$ myoblasts and differentiated myotubes (73). Our results show that Pak1 level is increased by $\sim 1.5$ fold in rat p67-expressing myoblasts and its conserved D251 and H331 residues along with its $\mathrm{N}$-terminal acidic residue-rich domain are involved in this process. On the other hand, the level of Pak1 is $\sim 3.0$-fold higher in rat p67-expressing myotubes and its $\mathrm{N}$-terminal acidic residue-rich domain but not the lysine-rich domains $1 \&$ II is involved in this process. P67 is also involved in the increased level of Cdc42 by proteolytic processing of the "pro" form of this protein, which is the upstream activator of both group A and group B Paks (73). Together, these results suggest that p67 is involved in the upregulated expression and activity of Pak1 in $\mathrm{C} 2 \mathrm{C} 12$ myotubes and thus it may be involved in the control of cytoskeleton dynamics including survival, migration, and motility of differentiated $\mathrm{C} 2 \mathrm{C} 12$ myoblasts (73). The level of p67 gradually increases while $\mathrm{C} 2 \mathrm{C} 12$ myoblasts were differentiating into myotubes and peaks at the time of myoblasts' fusion into myotubes (32). P67's direct or indirect effect on increased levels of Pak1 expression in myotubes indicates that it may be involved in other processes like motility of myotubes and control of glucose metabolism in the myotubes through the regulation of expression of Pak1. In addition to its role in expression of Pak1, p67 is involved in the maturation of "pro" form of Cdc42 to increase the level of this protein (73), which is the upstream effector of Pak1, indicates that p67 may be involved in the regulation of Pak1 activity as well. By regulating the expression activity of Pak1 p67 is possibly involved in motility and glucose metabolism in myotubes. 


\section{P67 IS INVOLVED IN THE INHIBITION OF INTEGRIN/FAK-MEDIATED SIGNALING INTO C2C12 MYOBLASTS DIFFERENTIATING INTO MYOTUBES}

Proliferation and differentiation of cells are regulated by extracellular signals from growth factors and extracellular matrix (ECM) proteins $(34,74)$. The Integrin-mediated adhesion to ECM controls proliferation and differentiation of myoblasts (75). Upon Integrin binding to ECM proteins, focal adhesion kinase (FAK) is activated and interacts with several downstream effectors to propagate signals that regulate cellular processes such as proliferation, migration, survival, and differentiation (76-77). Both Integrin and FAK are essential for fusion of myoblasts into myotubes $(10,78)$. In fact, during fusion of myoblasts FAK is transiently upregulated and possibly proteolytically degraded either in the proteasome or by cellular proteinases like calpain and others (79). This degradation is essential to inhibit the activity of FAK, which has strong growth promoting effects in many cells including almost all tumor cells (80).

A cytoplasmic protein tyrosine kinase, FAK is over-expressed and activated in several advancedstage solid tumors (80). FAK activation involves integrin receptor clustering upon cell binding to ECM proteins, which may involve FAK dimerization followed by auto-phosphorylation at Y397. Srcfamily of kinases binds to this phosphorylated site and forms an activated FAK-Src complex $(80,82$ 83). This activation promotes cell movement, invasion, motility, survival, and proliferation through kinase-dependent and -independent mechanisms (80-82). During myogenesis, FAK has been shown to be degraded at the proteasome (79). It is also degraded by other proteinases like calpain (79). Given the facts that FAK has a strong growth promoting signals that lead to tumorigenesis in almost all tissues and growth inhibition of $\mathrm{C} 2 \mathrm{C} 12$ myoblasts are essential to withdraw permanently from cell cycle for their fusion into myotubes, it imperative to degrade FAK during myogenesis.

P67 has both intermolecular and intra-molecular proteolysis activity (84) and therefore we examined whether p67 has any role in degrading FAK during the fusion of $\mathrm{C} 2 \mathrm{C} 12$ myoblasts into myotubes. We found that degradation of FAK peaked at the time of fusion of $\mathrm{C} 2 \mathrm{C} 12$ myoblasts into myotubes and at the same time the level of p67 is also high. In addition, p67's N-terminal lysine-rich domains I \& II and acidic residue-rich domain are involved in this degradation of FAK possibly by increasing the cellular concentration of p52 segment of p67 that has intermolecular proteolysis activity. We also found that the level of the Akt1/2 kinase, one of the several downstream effectors of FAK, gradually increased during the differentiation of $\mathrm{C} 2 \mathrm{C} 12$ myoblasts, possibly to prevent differentiated $\mathrm{C} 2 \mathrm{C} 12$ myoblasts from apoptosis and this process may be independent of FAK. During differentiation, $\mathrm{C} 2 \mathrm{C} 12$ myoblasts fuse into multinucleated myotubes and p67's level peaks at the same time of fusion (32). In this study, we noticed the increased degradation of FAK and possibly its inactivation at the time of myoblasts' fusion into myotubes and over-expression of rat p67 mutants such D6/2 and K1K2 in rat tumor hepatoma cells degraded FAK completely (85). These results therefore suggest that p67's $\mathrm{N}$-terminal acidic residue-rich domain and lysine-rich domains I \& II are involved in this FAK degradation process. Mutation to these domains possibly increases the cellular concentration of p52 segment of p67 and that in term may have intermolecular proteolysis activity, which degrades FAK to allow $\mathrm{C} 2 \mathrm{C} 12$ myoblasts fuse into multinucleated myotubes. We also found that the level of the Akt1/2 kinase, one of the several downstream effectors of FAK, gradually increased during the differentiation of $\mathrm{C} 2 \mathrm{C} 12$ myoblasts, possibly to prevent differentiated $\mathrm{C} 2 \mathrm{C} 12$ myoblasts from apoptosis and this process may be independent of FAK.

\section{CONCLUDing REMARKS}

A limited number of myogenic satellite cells or myoblasts are always present in contractile myofibers. Their numbers increase in injured tissues with the help of adequate blood supply that provides growth-promoting signal(s) for cell division. These cycling myoblasts are then programmed to express appropriate myogenic-specific proteins. In promoters of almost all myogenic genes there is more than one cis-element for binding to myogenic enhancer factor 2 (MEF2) that activates their expression in myoblasts and myotubes. These myogenic proteins then orchestrate the whole myogenic sequences in multiple steps that lead the myoblasts to fuse into multinucleated myotubes and ultimately these myotubes align to form contractile myofibers. One such myogenic protein, p67, which not only associates with eIF2 to regulate the rates of global protein synthesis, it also binds to ERK1/2 kinases to modulate their activation and activity to inhibit the growth-promoting signal mediated by the later kinases when needed by the cells. There are nine MEF2 cis-elements in mouse p67 promoter, two in rat promoter, and four in human promoter (86). Indeed, the expression of p67 
increases by several folds during differentiation of mouse $\mathrm{C} 2 \mathrm{C} 12$ myoblasts into myotubes. During differentiation, p67 dissociates from eIF2 and associates with ERK1/2 kinases. P67's dissociation from eIF2 leads to increase phosphorylation of eIF2 $\alpha$ that causes the suppression of global rates of protein synthesis (26) whereas, it association with ERK1/2 kinases leads to the inhibition of activation and activity of the later kinases to suppress the growth-promoting signal to myoblasts $(32,35)$ and allow these myogenic cells to withdraw permanently from the cell cycle and enter into the quiescence stage. For the later stage, degradation of several cyclin/Cdk complexes is absolutely necessary. Increased level of p67 in myoblasts and myotubes is involved in the expression and degradation of these cyclin/Cdk complexes (41). Involvement of p67's proteolysis activity is not limited to the degradation of cyclin/Cdk complexes it is also involved in the degradation of MARCKS and FAK that provide potent growth-promoting signals to myoblasts $(66,85)$. Increased levels of these proteins inhibit actin cytoskeleton dynamics, migration, and alignment of myoblasts while fusing into myotubes. By degrading these later proteins p67 helps myoblasts to differentiate into myotubes. In addition, p67 possibly helps differentiated myoblasts to maintain glucose metabolism and motility by upregulating the expression and activity of Pak1 (73), which is involved in the glucose homeostasis both in murine and human. Overexpression of p67 in C2C12 myoblasts increases the levels of cPKC $\alpha$ significantly and this increased level has the ability to differentiate myogenic cells into myotubes even in the presence of serum that supplies growth factors to cells for division. The later event is the reminiscence of the conditions when myoblasts multiple, migrate, align on the top of exiting wounded myotubes, and fuse into it to regenerate muscles during wound healing. Mouse $\mathrm{C} 2 \mathrm{C} 12$ myoblasts have been used as an excellent ex vivo model system to study molecular details of various multi-steps myogenic processes and differentiation of human skeletal myoblasts share many of these molecular details (87). Rhabdomyosarcoma is a human disease associated with the lack of differentiation of human satellite myogenic cells (88-89). Studying the detailed molecular mechanisms of p67's involvement in the multi-step differentiation processes during myoblasts' fusion into myotubes will greatly help our understanding of the above human deadly disease and its possible cure in the foreseeable future.

\section{REFERENCES}

[1] Buckingham, M., and Rigby, P. W. J. (2014) Gene regulatory networks and transcriptional mechanisms that control myogenesis. Dev. Biol. 28, 225-238.

[2] Potthoff, M. J. and Olson, E. N. (2007) MEF2: a central regulator of diverse developmental programs. Development 134, 4131-4140.

[3] Duronio, R. J. and Xiong, Y. (2013) Signaling pathways that control cell proliferation. Cold Spring Harb Perspect Biol. 5, 1-12.

[4] Dedieu, S., Poussard, S., Mazeres, G., Grise, F., Dargelos, E., Cottin, P., and Brustis, J.-J. (2004) Myoblast migration is regulated by calpain through its involvement in cell attachment and cytoskeleton organization. Exp. Cell. Res. 292, 187-200.

[5] Pipes, G. C. and Creemers, E. E., and Olson, E. N. (2006) The myocardin family of transcriptional coactivators: versatile regulators of cell growth, migration, and myogenesis. Genes Dev. 20, 1545-1556.

[6] Dedieu, S., Mazeres, G., Poussard, S., Brustis, J.-J., and Cottin, P. (2003) Myoblast migration is prevented by a calpain-dependent accumulation of MARCKS. Biol. Cell, 95, 615-623.

[7] Mazeres, G., Leloup, L., Daury, L., Cottin, P., and Brustis, J.-J. (2006) Myoblast attachment and spreading are regulated by different patterns by ubiquitous calpains. Cell Motility and the Cytoskeleton 63, 193-207.

[8] Suetsugu, S. (2010) The proposed functions of membrane curvatures mediated by the BAR domain superfamily proteins. J. Biochem. 148, 1-12.

[9] Sastry, S. K., Lakonishok, M., Wu, S., Truong, T. Q., Huttenlocher, A., Turner, C. E., and Horwitz, A. F. (1999) Quantitative changes in integrin and focal adhesion signaling regulate myoblast cell cycle withdrawal. J. Cell Biol. 144, 1295-1309.

[10] Quach, N. I., Biressi, S., Reichardt, L. F., Keller, C., and Rando, T. A. (2009) Focal adhesion kinase signaling regulates the expression of caveolin 3 and betal integrin, genes essential for normal myoblast fusion. Mol. Biol. Cell 20, 3422-3435. 
[11] Abmayr, S. M., and Pavlath, G. K. (2012) Myoblast fusion: lessons from flies and mice. Development 139, 641-656.

[12] Bassel-Duby, R. and Olson, E. N. (2003) Role of calcineurin in striated muscle: development, adaptation, and disease. Biochem. Biophys. Res. Comm. 311, 1133-1141.

[13] Kumar, K. S., Ramadhas, A., Nayak, S. C., Kaniyappan, S., Dayma, K., and Radha, V. (2015) C3G (RapGEF1), a regulator of actin dynamics promotes survival and myogenic differentiation of mouse mesemchymal cells. Biochim. Biophys. Acta 1853, 2629-2639.

[14] Mitra, S. K., Hanson, D. A., and Schlaepfer, D. D. (2005) Focal adhesion kinase: in command and control of cell motility. Nat. Rev. Mol. Cell Biol. 6, 56-68.

[15] Gundersen, K. (2011) Excitation-transcription coupling in skeletal muscle: the molecular pathways of exercise. Biol. Rev. 86, 564-600.

[16] Aurora, A. B. and Olson, E. N. (2014) Immune modulation of stem cells and regeneration. Cell Stem Cell. 15, 14-25.

[17] Zembron-Lacny, Krywanski, J., Ostapiuk-Karolczuk, J., and Kasperska, A. (2012) Cellular and molecular mechanisms of regeneration and remodeling of skeletal muscles. Ortop. Traum. Rehab. 14, 1-11.

[18] Karalaki, M., Philippou, A., and Koutsilieris, M. (2009) Muscle regeneration: cellular and molecular events. In vivo 23, 779-796.

[19] Asp, P., Blum, R., Vethantham, V., Parisi, F., Micsinai, M., Cheng, J., Bowman, C., Kluger, Y., and Dynlacht, B. D. (2011) Genome-wide remodeling of the epigenetic landscape during myogenic differentiation. Proc. Natl. Acad. Sci. USA 108, E149-E158.

[20] Gaboardi, G. C., Ramazzotti, G., Bavelloni, A., Piazzi, M., Fiume, R., Billi, A. M., Matteucci, A., Faenza, I., and Cocco, L. (2010) A role for PKC $\varepsilon$ during $\mathrm{C} 2 \mathrm{C} 12$ myogenic differentiation. Cell. Signalling 22, 629-635.

[21] Raman, M., Chen, W., and Cobb, M. H. (2007) Differential regulation and properties of MAPKs. Oncogene 26, 3100-3112.

[22] Biro, T., Czifra, G., Bodo, E., Lazar, J., Papp, H., Kovacs, I., Juhasz, I., and Kovacs, L. (2004) Cell and isoform specific roles of protein kinase $\mathrm{C}$ isozymes in regulating in vitro and in vivo proliferation of keratinocytes and skeletal muscle cells. J. Invest. Dermatol. 122, A21.

[23] Datta, B. (2009) Roles of P67/MetAP2 as a tumor suppressor - a review. Biochim. Biophys. Acta 1796, 281-292.

[24] Datta, B. (2015) Diversified roles of p67/MetAP2 as a regulator of cell growth and differentiation, in tumor suppression, and in obesity. Curr. Topics Biochem. Res. 16, 41-52.

[25] Datta, B., Chakrabarti, D., Roy, A.L., and Gupta, N.K. (1988) Roles of a 67kDa polypeptide in protein synthesis inhibition in heme-deficient reticulocyte lysates. Proc. Natl. Acad. Sci. USA 85, 3324-3328.

[26] Datta, B. and Datta, R. (2014) Phosphorylation of eukaryotic initiation factor $2 \alpha$ during differentiation of mouse myoblasts into myotubes is mediated by an unknown kinase. Intl. $J$. Adv. Res. Chem. Sci. 1, 1-5.

[27] Datta, R., Choudhury, P., Bhattacharya, M., Leon, F. S., Zhou, Y., and Datta, B. (2001) Protection of translation initiation factor eIF2 phosphoryaltion correlates with eIF2-associated glycoprotein p67 levels and requires the lysine-rich domain I of p67. Biochimie 83, 919-931.

[28] Datta, R., Tammali, R., and Datta, B. (2003) Negative regulation of the protection of eIF2 $\alpha$ phosphorylation activity by a unique acidic domain present at the N-terminus of p67. Exp. Cell Res. 283, 237-246.

[29] Datta, B. and Datta, R. (2003) Mutation at the acidic residue-rich domain of eukaryotic initiation factor 2 (eIF2 $\alpha$ )-associated glycoprotein p67 increases the protection of eIF2 $\alpha$ phosphorylation during heat shock. Arch. Biochem. Biophys. 413, 116-122.

[30] Datta, R., Choudhury, P., Ghosh, A., and Datta, B. (2003) A glycosylation site, ${ }_{60} \mathrm{SGTS}_{63}$, of p67 is required for its ability to regulate the phosphorylation and activity of eukaryotic initiation factor2 alpha (eIF2 $\alpha$ ). Biochemistry 42, 5453-5460. 
[31] Datta, B., Datta, R., Majumdar, A., and Balusu, R. (2004) Treatment of cells with the angiogenic inhibitor, fumagillin results in increased stability of eukaryotic initiation factor 2 -associated glycoprotein, p67, and reduced phosphorylation of extra-cellular signal-regulated kinases 1 and 2. Biochemistry 43, 14821-14831.

[32] Datta, B., Datta, R., Majumdar, A., and Ghosh, A. (2005) The stability of eukaryotic initiation factor 2-associated glycoprotein, p67, increases during skeletal muscle differentiation and that inhibits the phosphorylation of extracellular signal-regulated kinases 1 and 2. Exp. Cell Res. 303, 174-182.

[33] Torii, S., Yamamoto, T., Tsuchiya, Y., and Nishida, E. (2006) ERK MAP kinase in G cell cycle progression and cancer. Cancer Sci. 97, 697-702.

[34] Meloche, S., and Pouyssegur, J. (2007) The ERK1/2 mitogen-activated protein kinase pathway as a master regulator of the G1- to S-phase transition. Oncogene 26, 3227-3239.

[35] Majumdar, A., Ghosh, A., Datta, S., Prudner, B., and Datta, B. (2010) P67/MetAP2 suppresses K-RasV12 mediated transformation of NIH3T3 mouse fibroblasts in culture and in athymic mice. Biochemistry 49, 10146-10157.

[36] Deschenes-Simard, X., Kottakis, F., Meloche, S. and Ferbeyre, G. (2014) ERKs in cancer: Friends or Foes? Cancer Res. 74, 412-419.

[37] Katz, M., Ido, A., and Yarden, Y. (2007) Regulation of MAPKs by growth factors and receptor tyrosine kinases. Biocheim. Biophys. Acta 1773, 1161-1176.

[38] Malumbres, M. (2014) Cyclin-dependent kinases. Genome Biol. 15, 122-131.

[39] Lim, S., and Kaldis, P. (2013) Cdks, cyclins and CKIs: roles beyond cell cycle regulation. Development 140, 3079-3093.

[40] Benanti, J. A. (2012) Coordination of cell growth and division by the ubiquitin-proteasome system. Semin. Cell Dev. Biol. 23, 492-498.

[41] Datta, S. K. and Datta, B. (2016) P67 shows differential effects in proteolysis and maturation of cell cycle regulatory proteins, cyclins and Cdks. Intl. J. Adv. Res. Chem. Sci. 3, 1-10.

[42] Schmitz-Peiffer, C. (2013) The tail wagging the dog - regulation of lipid metabolism by protein kinase C. FEBS J. 280, 5371-5383.

[43] Kang, J.-H. (2014) Protein kinase C (PKC) isozymes and cancer. New. J. Sci. 1, 1-36.

[44] Garg, R., Benedetti, L. G., Abera, M. B., Wang, H., Abba, M., Kazanietz, M. G. (2014) Protein kinase $\mathrm{C}$ and Cancer: what we know and what we do not. Oncogene 33, 5225-5237.

[45] Kang, J.-H., Asai, D., Toita, R., Kitazaki, H., and Katayama, Y. (2009) Plasma protein kinase C $(\mathrm{PKC} \alpha)$ as a biomarker for diagonosis of cancers. Carcinogenesis, Vol. 30, pp. 1927-1931.

[46] Kang, J.-H., Mori, T., Kitazaki, H. et al. (2013) Kinase activity of protein kinase C $\alpha$ in serum as a diagnostic biomarker of human lung cancer. Anticancer Res. 33, 485-488.

[47] Kang, J.-H., Mori, T., Kitazaki, H. et al. (2013) Serum protein kinase C $\alpha$ in serum as a diagnostic biomarker of cancers. Cancer Biomarkers, 13, 99-103.

[48] Assender, J. W., Gee, J. M. W., Lewis, I., Ellis, I. O., Robertson, J. F. R., and Nicholson, R. I. (2007) protein kinase $\mathrm{C}$ isoform expression as a predictor of disease outcome on endocrine therapy in breast cancer. J. Clin. Path. 60, 1216-1221.

[49] Lonne, G. K., Cornmark, L., Zahirovic, I. O., Landberg, G., Jirstrom, K., and Larsson, C. (2010) PKC $\alpha$ expression is a marker for breast cancer aggressiveness. Mol. Cancer 9, article 76.

[50] Czifra, G., Szollosi, A., Nagy, Z., Boros, M., Juhasz, I., Kiss, A., Erdodi, F., Szebo, T., Kivacs, I., Torok, M., Kovacs, L., Blumberg, P. M., and Biro, T. (2015) Protein kinase C $\delta$ promotes proliferation and induces malignant transformation in skeletal muscle. J. Cell. Mol. Med. 19, 396-407.

[51] Meacci, E., Donati, C., Cencetti, F., Romiti, E., and Bruni, P. (2000) Permissive role of protein kinase $\mathrm{C} \alpha$ but not protein kinase $\mathrm{C} \delta$ in sphingosine 1-phosphate-induced RhoA activation in C2C12 myoblasts. FEBS let. 482, 97-101.

[52] Czifra, G., Toth, I. B., Marincsak, R., Juhasz, I., Kovacs, I., Acs, P., Kivacs, L., Blumberg, P. M., and Biro, T. (2006) Insulin-like growth factor-1-coupled mitogen signaling in primary cultured human skeletal muscle cells and in $\mathrm{C} 2 \mathrm{C} 12$ myoblasts. A central role of protein kinase Co. Cell. Signalling 18, 1461-1472. 
[53] Selvaraj, A., and Prywes, R. (2003) Megakaryoblastic keukemia-1/2, a transcriptional coactivator of serum response factor, is required for skeletal myogenic differentiation. J. Biol. Chem. 278, 41977-41987.

[54] Iyer, D., Chang, D., Marx, J., Wei, L., Olson, E. N., Parmacek, M. S., Balasubramanyam, A., and Schwartz, R. J. (2006) Serum response factor MADS box serine-162 phosphorylation and myogenic gene programs. Proc. Natl., Acad. Sci. USA. 103, 4516-4521.

[55] Fedorov, Y. V., Jones, N. C., and Olwin, B. B. (2002) Atypical protein kinase Cs are the Ras effectors that mediate repression of myogenic satellite cell differentiation. Mol. Cell. Biol. 22, 1140-1149.

[56] Chappell, D. S., Patel, N. A., Jiang, K., Li, P., Watson, J. E., Byers, D. M., and Cooper, D. R. (2009) Functional involvement of protein kinase C- $\beta$ II and its substrate, myristoylated alaninerich C-kinase substrate (MARCKS), in insulin-stimulated glucose transport in L6 rat skeletal muscle cells. Diabetologia, 52, 901-911.

[57] Taniguchi, H., Manenti, S., Suzuki, M., and Titani, K. (1994) Myristoylated alanine-rich C kinase substrate (MARCKS), a major protein kinase $\mathrm{C}$ substrate, is an in vivo substrate of proline-directed protein kinase(s). A mass spectroscopic analysis of the post-translational modifications. J. Biol. Chem. 269, 18299-18302.

[58] Herget, T., Oehrlein, S. A., Pappin, D. j. C., Rozengurt, E., and Parker, P. J. (1995) The myristoylated alanine-rich C-kinase substrate (MARCKS) is sequentially phosphorylated by conventional, novel and atypical isotypes of protein kinase C. Eur. J. Biochem., 233, 448-457.

[59] Dedieu, S., Mazeres, G., Poussard, S., Brustis, J.-J., and Cottin, P. (2003) Myoblast migration is prevented by a calpain-dependent accumulation of MARCKS. Biol. Cell, 95, 615-623.

[60] Dedieu, S., Poussard, S., Mazeres, G., Grise, F., Dargelos, E., Cottin, P., and Brustis, J.-J. (2004) Myoblast migration is regulated by calpain through its involvement in cell attachment and cytoskeleton organization. Exp. Cell. Res. 292, 187-200.

[61] Brudvig, J. J. and Weimer, J. M. (2015) X MARCKS the spot: myristoylated alanine-rich C kinase substrate in neuronal function and disease. Frontiers in Neurscience, 9, 1-10.

[62] Mazeres, G., Leloup, L., Daury, L., Cottin, P., and Brustis, J.-J. (2006) Myoblast attachment and spreading are regulated by different patterns by ubiquitous calpains. Cell Motility and the Cytoskeleton 63, 193-207.

[63] Louis, M., Zanou, N., Schoor, M. V., and Gailly, P. (2008) TRPC1 regulates skeletal myoblast migration and differentiation. J. Cell Sci. 121, 3951-3959.

[64] Kim, S. S., Kim, J. H., Lee, S.-H., Chung, S. S., Bang, O.-S., Park, D., and Chung, C. H. (2002) Involvement of protein phosphatase-1-mediated MARCKS translocation in myogenic differentiation of embryonic muscle cells. J. Cell Sci. 115, 2465-2473.

[65] Kim, S. S., Kim, J. H., Kim, H. S., Park, D. E., and Chung, C. H. (2000) Involvement of the $\theta-$ type protein kinase $\mathrm{C}$ in translocation of myristoylated alanine-rich $\mathrm{C}$ kinase substrate (MARCKS) during myogenesis of chick embryonic myoblasts. Biochem. J. 347, 139-146.

[66] Datta, S. K. and Datta, B. (2016) Differential expression of MARCKS in C2C12 myoblasts and myotubes constitutively expressing p67. Int. J. Adv. Res. Chem. Sci. 3(2), 15-22.

[67] Rane, C. K., and Minden, A. (2014) P21 activated kinases: structure, regulation, and functions. Small GTPases, 5, e28003.

[68] Etienne-Manneville, S. (2004) Cdc42 - the centre of polarity. J. Cell. Sci. 117, 1291-1300.

[69] Dummler, B., Ohshiro, K., Kumar, R., Field, J. (2009) Pak protein kinases and their role in cancer. Cancer Metastasis Rev. 28, 51-63.

[70] Buss, F., Kendrick-Jones, J., Lionne, C., Knight, A. E., Core, G. P., and Paul Luzio, J. (1998) The localization of myosin VI at the golgi complex and leading edge of fibroblasts and its phosphorylation and recruitment into membrane ruffles of A431 cell after growth factor stimulation. J. Cell. Biol. 143, 1535-1545.

[71] Ye, D. Z., and Field, J. (2012) PAK signaling in cancer. Cell Logist 2, 105-116.

[72] Wang, Z., Oh, E., Clapp, D. W., Chernoff, J., and Thurmond, D. C. (2011) Inhibition or ablation of p21-activating kinase (PAK1) disrupts glucose homeostatic mechanisms in vivo. J. Biol. Chem. 286, 41359-41367. 
Involvement of p67 in Actin Cytoskeleton Dynamics, Survival, Migration, and Motility during Differentiation of C2C12 Myoblasts into Multinucleated Myotubes

[73] Datta, B. and Datta, R. (2016) P67 is involved in the regulation of cytoskeleton dynamics by modulating the expression and activity of Pak1 in differentiated $\mathrm{C} 2 \mathrm{C} 12$ myoblasts. Int. J. Adv. Res. Chem. Sci. 3(4), 1-8.

[74] Han, J. W., Lee, H.-J., Bae, G.-U., and Kang, J.-S. (2011) Promyogenic function of integrin/FAK signaling is mediated by Cdo, Cdc2, and MyoD. Cellular Signalling 23, 1162-1169.

[75] Sastry, S. K., Lakonishok, M., Wu, S., Truong, T. Q., Huttenlocher, A., Turner, C. E., and Horwitz, A. F. (1999) Quantitative changes in integrin and focal adhesion signaling regulate myoblast cell cycle withdrawal. J. Cell Biol. 144, 1295-1309.

[76] Schaller, M. D. (2001) Biochemical signals and biological responses elicited by the focal adhesion kinase. Bichim. Biophys. Acta 1540, 1-21.

[77] Schlaepfer, D. D., and Mitra, S. K. (2004) Multiple connections link FAK to cell motility and invasion. Curr. Opin. Genet. Dev. 14, 92-101.

[78] Clemente, C. F. M. Z., Corat, M. A. F., Saad, S. T. O., and Franchini, K. G. (2005) Differentiation of $\mathrm{C} 2 \mathrm{C} 12$ myoblasts is critically regulated by FAK signaling. Am. J. Physiol. Regul. Integr. Comp. Physiol. 289, R862-R870.

[79] Mitra, S. K., Hanson, D. A., and Schlaepfer, D. D. (2005) Focal adhesion kinase: in command and control of cell motility. Nat. Rev. Mol. Cell Biol. 6, 56-68.

[80] Sulzmaier, F. J., Jean, C., and Schlaepfer, D. D. (2015) FAK in cancer: mechanistic findings and clinical applications. Nat. Rev. Cancer 14, 598-610.

[81] Clark, A. R. and Toker, A. (2014) Signaling specificity in the Akt pathway in breast cancer. Biochem. Soc. Trans. 42, 1349-1355.

[82] Schaller, M. D. (2010) Cellular functions of FAK kinases: insight into molecular mechnisms and novel functions. J. Cell Sci. 123, 1007-10013.

[83] Hoskin, U., Szeto, A., Ghaffari, A., Greer, R. A., Cote, G. P., and Elliott, B. E. (2015) Ezrin regulates focal adhesion and invadopodia dynamics by altering calpain activity to promote breast cancer cell invasion. Mol. Biol. Cell 26, 3464-3479.

[84] Datta, B. (2016) Intra-molecular and Intermolecular Proteolysis Activity of p67. Int. J. Adv. Res. Chem. Sci. (In Press).

[85] Datta, B. and Datta, R. (2016) The N-terminal acidic residue-rich domain and lysine-rich domains I and II of p67 are required for stable expression of focal adhesion kinase (FAK). Int. J. Adv. Res. Chem. Sci. 3(3), 1-6.

[86] Datta, B., Earl, D., Roods, M., Datta, S. (2014) Analysis of p67/MetAP2 gene from mouse, rat, and human. Intl. J. Mol. Genet. 5, 1-12.

[87] Osterloh, M., Bohm, M., Kalbe, B., Osterloh, S., and Hatt, H. (2015) Identification and functional characterization of TRPA1 in human myoblasts. Pflugers Arch-Eur. J. Physiol. 15, 1729-1741.

[88] Sun, X., Gua, W., Shen, J. K., Mankin, H. J., Hornicek, F. J., and Duan, Z. (2015) Rhabdomyosarcoma: Advances in Molecular and Cellular Biology. Sarcoma, Article ID 232010, $1-14$.

[89] Hettmer, S., Li, Z., Billin, A. N., Barr, F. G., Cormelison, D. D. W., Ehrlich, A. R., Guttridge, D. C., Hayes-Jordan, A., Helman, L. J., Houghton, P. J., Khan, J., Langenau, D. M., Linardic, C. M., Pal, R., Partridge, T. A., Pavlath, G. K., Rota, R., Schafer, B. W., Shipley, J., Stillman, B., Wexler, L. H., Wagers, A. J., and Keller, C. (2014) Cold Spring Harb. Perspect. Med. 4, 1-8.

\section{AUTHOR'S BIOGRAPHY}

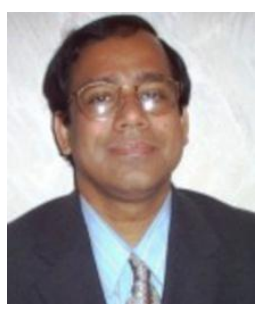

Bansidhar Datta, Ph.D., Associate Professor, Department of Chemistry \& Biochemistry, Kent State University, Kent, OH 44240, USA 